\title{
NONSYNDROMIC OLIGODONTIA: A RARE CASE REPORT WITH REVIEW OF LITERATURE
}

Puneet Bajaj $^{1}$, Robin Sabharwal' ${ }^{2}$, Sonia Joshi ${ }^{3}$

${ }^{1}$ Prof. \& Head, Department of Oral Pathology and Microbiology, Bhojia Dental College and Hospital, Baddi, Himachal Pradesh.

${ }^{2}$ Sr.Lecturer, Department of Oral Pathology and Microbiology, Bhojia Dental College and Hospital, Baddi, Himachal Pradesh

${ }^{3}$ Post Graduate Student, Oral Pathology and Microbiology,Bhojia Dental College and Hospital, Baddi, Himachal Pradesh

Corresponding Author: Sonia Josh Mobile: +91- 973680763 E-mail : soniajoshiop@gmail.com

Received:29 $9^{\text {th }}$ April 2014 Accepted: $26^{\text {th }}$ July 2014 Online: $20^{\text {th }}$ Sept 2014

ABSTRACT

Dental agenesis is the most common developmental anomaly in humans and is frequently associated with several other oral abnormalities. In the literature, some terms are used to describe missing teeth like Oligodontia, Anodontia and Hypodontia. Oligodontia is defined as the developmental absence of six teeth or more, excluding third molars. It can be isolated or as a part of a syndrome such as in ectodermal dysplasia. The present case describes agenesis of permanent teeth which are non-familial and with no apparent systemic abnormalities

Key words: Agenesis, Anodontia, Developmental Anomalies, Hypodontia, Oligodontia, Oral Abnormalities.

\section{Introduction}

Dental agenesis is the most common developmental anomaly in humans, often presenting a significant clinical problem. It is classified according to the number of missing permanent teeth excluding the third molars. ${ }^{1}$ Hypodontia is used to describe the absence of one or few teeth, Oligodontia is used for agenesis of numerous teeth (more than six teeth) excluding the third molars and anodontia is the extreme of oligodontia where there is total absence of any dental structure. ${ }^{2}$ Oligodontia is also known as partial anodontia, severe or advance anodontia. ${ }^{3}$ Some of them also refer this as selective tooth agenesis. ${ }^{4}$ According to different authors, the frequency of hypodontia is 1$10 \%$ and oligodontia $0.1-0.9 \% .^{5,6}$ Anodontia occurs very rarely (17 cases were described over the last 50 years). Oligodontia may occur as a part of a genetic syndrome, as a non syndromic isolated familial trait, as an infrequent finding or as an isolated condition that has been linked to mutations of the MSX1 and PAX9. ${ }^{8}$

The list of all the genes and molecular pathways involved in tooth agenesis are shown in Table 1.
In addition to genetic factors, the congenital absence of teeth may result from disturbances during the initial stages of tooth development such as trauma, radiation, metabolic disorders, localized inflammation, infectious disease such as

Table 1: List of all the genes and molecular pathways involved in tooth agenesis

Wnt/b-catenin/LEF1
MSX1
MSX2
SHH
P63
Pitx2
Runx2Cbfa 1

Hypodontia and oligodontia are classified as isolated or non-syndromic hypodontia/ oligodontia and syndromic hypodontia/ oligodontia or hypodontia/ oligodontia associated with syndromes. ${ }^{11,12}$ Oligodontia can occur in association with genetic syndromes, such as ectodermal dysplasia, Incontinentia pigmenti, Down syndrome and Rieger syndrome or as a non-syndromic isolated familial trait, or as a sporadic finding. When oligodontia is associated with a syndrome there may be abnormalities of the skin, nails, eyes, ears and skeleton, while anodontia is 
commonly seen in severe cases of ectodermal dysplasia. $^{3,13,14}$

Congenital absence of teeth may be due to from physical obstruction or disruption of the dental lamina, space limitation and functional abnormalities of the dental epithelium or failure of initiation of the underlying mesenchyme. ${ }^{11}$ Characteristic dental symptoms are reduced number of teeth, a reduction in tooth size, anomalies of tooth form and delayed eruption. The absence of teeth in young patients can cause esthetic, functional and psychological problems particularly if the teeth of the anterior region are involved. ${ }^{12}$

The purpose of this article is to report a case of congenitally missing permanent teeth and thus the diagnosis of hypodontia/Oligodontia should be performed as early as possible in order to prevent aesthetic and functional problems in dentition.

\section{CASE REPORT}

A 4 year old girl patient reported to the department of Oral and Maxillofacial Pathology with the chief complaint of missing teeth and wanted replacement of
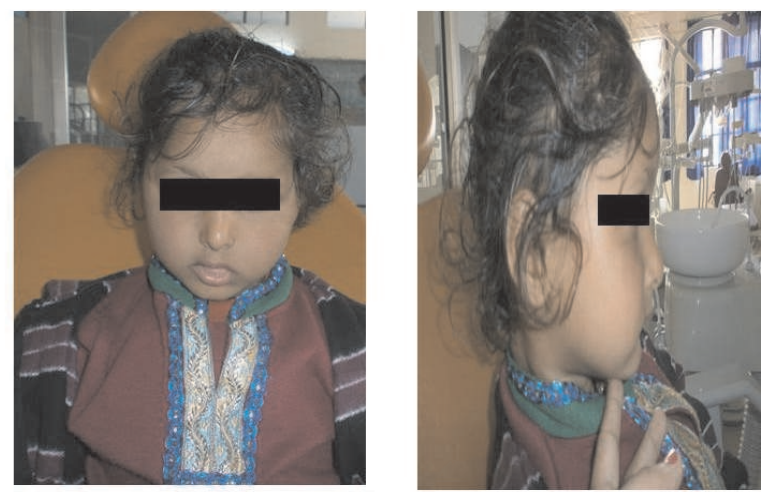

Figure 1: Extraoral photograph of 4 year old girl showing normal soft tissue
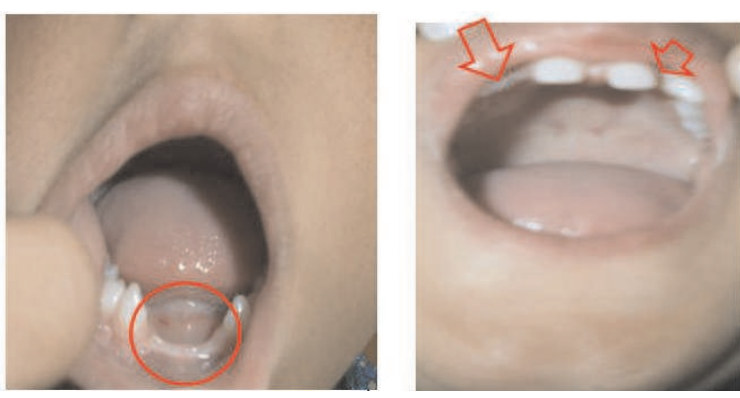

Figure 2: Intraoral photograph showing missing 52,62, 71, $72,81,82$. missing teeth. On examination, the patient's parents gave no significant past medical and dental history, neither there was any abnormality detected on general examination suggestive of any syndromes. The family history did not reveal any multiple missing teeth. Soft tissues were normal (Figure 1,2). Patient was born to non-consanguineous parents. There was no history of any infection or trauma.

Patient was healthy with no relevant medical and family history. Suspecting the congenital absence of permanent teeth panoramic radiograph was taken which showed presence of Fourteen fully erupted deciduous teeth along with presence of tooth buds of Fourteen permanent teeth $(11,21,13,23,26,36,17$, $27,33,36,37,43,46,47)$. Teeth present were 51,53 , $54,54,61,63,64,65,73,74,75,83,84,85$ (Figure 3). Queries revealed that missing six teeth like $52,62,71,72,81,82$ were not extracted and were absent since childhood. Also there was no developing tooth buds for Fourteen permanent teeth $(12,14,15$, $22,24,25,31,32,34,35,41,42,44,45)$ excluding the third molars. Also the developed tooth buds for permanent maxillary incisors $(11,21)$ were larger in size.

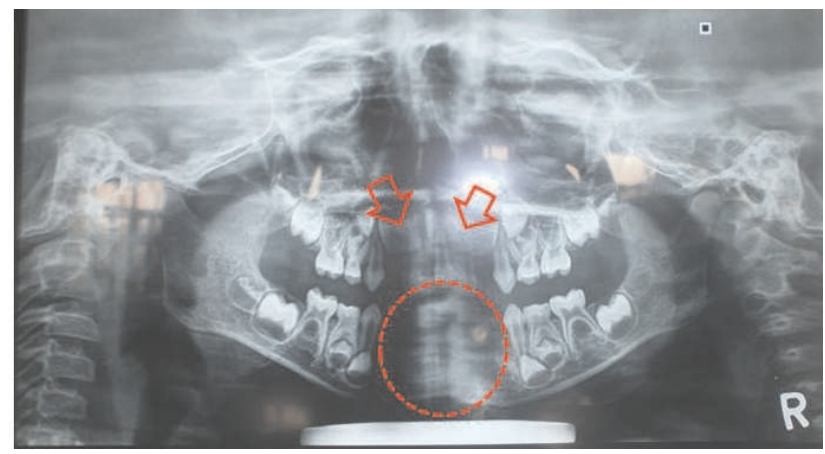

Figure 3: Panoramic radiograph showing missing teeth .

Based on clinical and radiological examinations, diagnosis of non syndromic oligodontia was made. The condition was explained to the patient and treatment plan was discussed.

\section{DISCUSSION}

Tooth agenesis, the congenital absence of one or more permanent teeth, is a common human anomaly. A tooth is defined to be congenitally missing if it has not erupted in the oral cavity and is not visible in a radiograph. ${ }^{12-14}$ 
In most populations, the reported prevalence of permanent tooth agenesis, excluding third molars, varies from 2.2 to $10.1 \%{ }^{15}$ In the majority of cases, persons are missing only one tooth. The prevalence becomes progressively smaller as the number of missing teeth increases. Agenesis of more than two teeth occurs in approximately $1 \%$ of the population. ${ }^{15}$

Selective tooth agenesis is divided into 2 types: hypodontia, the agenesis of fewer than 6 teeth, and oligodontia, the agenesis of six or more permanent teeth. In both cases, the third molars (wisdom teeth) are not included. Oligodontia is a rare anomaly, affecting approximately 0.1 to $0.3 \%$ of the population. ${ }^{15}$

According to a 1996 consensus conference on oral Implants in young patients, the following definitions are used; Hypodontia is defined as the absence of one to five permanent teeth, while the term oligodontia refers to the absence of six or more permanent teeth and 'anodontia' to the absence of all permanent teeth. ${ }^{16,14}$ Hypodontia and oligodontia are classified as isolated or non-syndromic hypodontia/oligodontia and syndromic hypodontia/oligodontia or hypodontia/oligodontia associated with syndromes. ${ }^{11}$ Most often oligodontia appears as part of some congenital syndromes that affect several organ systems. ${ }^{11}$ Oligodontia can occur in association with various genetic syndromes, such as ectodermal dysplasia, incontinentia pigmenti, Down syndrome, Rieger syndrome, Wolf- Hirschhorn syndrome, Van der Woude syndrome, Ectrodactyly- ectodermal dysplasia- clefting syndrome, Cleft lip palate, Ectodermal dysplasia syndrome, Oral facial digital syndrome type I, Witkop tooth-nail syndrome, Fried syndrome, Hair- nail- skin- teeth dysplasias, Hirschhorn syndrome, Hemifacial microsomia and Recessive incisor hypodontia. ${ }^{11,12}$

When oligodontia is associated with a syndrome there may be abnormalities of the skin, nails, eyes, ears and skeleton, which was not in the present case.

Oligodontia as well as hypodontia (lack of one or more permanent teeth) are highly heritable conditions associated with mutations in the AXIN2, MSX1, PAX9, EDA and EDAR genes. ${ }^{17}$ The incidence of oligodontia is reported to vary from $0.08-0.16 \%$. The pattern of tooth absence is influenced by the gene affected, as well as the type of mutations within the specific gene. ${ }^{18}$ The exact etiology for oligodontia is unknown. Various factors have been described in the literature. Heredity is the main etiological factor. ${ }^{14}$ Perhaps the best family study of tooth agenesis was done by Grahnen in $1956 .{ }^{19}$ He found that if either parent had one or more congenitally missing teeth, there was an increased likelihood that their children also would be affected. This familial relationship suggests that the genes are important but in our case family history was negative. ${ }^{12}$

Several environmental factors like virus infections, toxins and radio or chemotherapy may cause missing of permanent teeth, which was also not present present in our case as reported by parents.

Although several potential and verified environmental factors in tooth agenesis have been identified, genetic defects play a major role in the etiology. So far, researchers have identified genetic defects that cause tooth agenesis either as a sole anomaly (isolated or non-syndromic) or as a part of multiple congenital anomalies (syndromic). One gene associated with syndromic tooth agenesis is the EDA gene, which underlies X-linked hypohidrotic ectodermal dysplasia. Non-syndromic tooth agenesis has wide phenotypic heterogeneity and is classified as either sporadic or familial, which can be inherited in an autosomal-dominant, autosomal-recessive, or Xlinked mode. ${ }^{14,15}$

\section{CONCLUSION}

From detailed history and thorough clinical examination we came to the final diagnosis that this case is of non syndromic oligodontia.

Careful treatment planning and current understanding of rare conditions like tooth agenesis is important, because there is a need to deal with not only the immediate, but also the long- term adverse implications. Hence, multidisciplinary treatment planning that takes account of established and emerging techniques needs to be practiced. Patient with tooth agenesis may not only have functional problems but also psychological distress that requires early diagnosis and proper evaluation to improve oral health and psyche of the child.

\section{REFERENCES}

1. Mahadevi B, Puranik RS, Shrinivas S. Oligodontia: A Case report and review of literature. World J Dent 2011;2:259-62.

2. Gupta D, Kamal M, Sharma H, Gupta S. Idiopathic nonsyndromic oligodontia in permanent dentition: A Case Report. Indian J Dent Sci 2012;4:67-9.

3. Goya HA, Tanaka S, Maeda T, Akimoto Y. An orthopantimigraphic study of hypodontia in permanent teeth 
of Japanese pediatric patients. J Oral Sci 2008; 50:143-50.

4. Noor A, Windpassinger C, Vitcu I, Orlic M, Rafiq MA, Khalid $\mathrm{M}$, et al.Oligodontia is caused by mutation in LTBP3, the gene encoding Latent TGF-B Binding protein 3. Am J Hum Genet 2009;84:519-23

5. Biedziak B. Aetiology and occurrence of tooth agenesis: Review of the literature. Dent Med Probl 2004;41:531-5.

6. Jędryszek A, Kmiecik M, Paszkiewicz A. Review of modern knowledge on hypodontia. Dent Med Probl 2009;46:118-25.

7. Ohno K, Ohmori I. Anodontia with hypohydrotic ectodermal dysplasia in young female: A case report. Pediatr Den 2000;22:49-52.

8. Singer SL, Henry PJ, Lander ID. A Treatment Planning Classification For Oligodontia. Int J Prosthodont 2010;23:99106.

9. Brook AA. A Unifying aetiological explanation for anomalies of human tooth number and size Arch Oral Boil 1984;29:3738 .

10. Dali. Rare cases of idiopathic nonsyndromic tooth agenesis. Journal of Interdisciplinary Dentistry 2012;2:190-4.

11. Cakur B, Dagistan S, Milgolu and Bilge M. Nonsyndromic Oligodontia in Permanent Dentition: Three Siblings. The Internet Journal of Dental Science 2006;3:103-7.
12. Hiremath Mallayya C . Nonsyndromic Oligodontia: A Rare Case Report. AOSR 2012;2:103-7.

13. Hosur M, Puranik RS, Vanaki SS. Oligodontia: A Case Report and Review of Literature. World J Dent 2011; 2:25962.

14. Vijay kumar Biradar, Surekha Biradar. Non-Syndromic Oligodontia: Report of Two Cases and Literature Review . International Journal of Oral \& Maxillofacial Pathology. 2012;3:48-51

15. Suda N, Ogawa T, Kojima T, Saito C, Moriyama K. Nonsyndromic oligodontia with a Novel Mutation of PAX9. J Dent Res 2011;90:382-6.

16. Durstberger G, Celar A, Watzek G. Implant-surgical and prosthetic rehabilitation of patients with multiple dental aplasia: a clinical report. Int J Oral Maxillofac Implants 1999;14:417-23.

17. Bergendal B, Klar J, Stecksén-Blicks C, Norderyd J, Dahl N. Isolated oligodontia associated with mutations in EDARADD, AXIN2, MSX1 and PAX9 genes. Am J Med GenetA2011;155:1616-22.

18. Singer SL, Henry PJ, Lander ID. A treatment planning classification for oligodontia. Int J Prosthodont 2010;23:99106.

19. Grahnen H. Hypodontia in the permanent dentition. Clinical and genetical investigation. Odont Revy 1956;7:15-9.

Source of Support: Nil, Conflict of Interest: None Declared 